\title{
Playing by the Rules Using Decision Rules Wisely Part 1, Trauma
}

Biren A. Bhatt, MD; Emmagene Worley, MD

\section{In part 1 of this 2-part review, the authors discuss validated trauma-related clinical decision rules most commonly used in the ED, and provide useful pearls and pitfalls pertaining to their use.}

Dr Bhatt is an assistant clinical professor of medicine, department of emergency medicine, New York-Presbyterian Hospital, New York, New York. Dr Worley is a chief resident, department of emergency medicine, New York-Presbyterian Hospital, New York, New York.

Authors' Disclosure Statement: The authors report no actual or potential conflict of interest in relation to this article.

DOI: 10.12788/emed.2017.0073

ability of disease and to identify high-risk features that should prompt further investigation. The formation of clinical decision rules entails at least three steps, which Ian Stiell, MD, emergency medicine's (EM's) most prolific author of these instruments, describes as follows:

- Creation of the rule or derivation;

- Prospective assessment of the reliability, accuracy, and impact of the rule in a validation study; and

- Gauging the effect of the rule on patient care through an implementation study. ${ }^{1}$

In addition to these three steps, many clinicians argue that there should also be an important fourth step included in this process: the external validation or assessment of the rule outside of the original study site(s), to assure reliability of the rule across a variety of populations for which its use was intended. ${ }^{2}$
Clinical decision rules seek to decrease resource utilization in instances of low prob- 


\section{EMERGENCY}

\section{NEXUS}

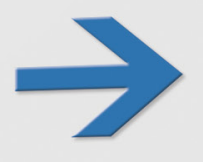

PECARN

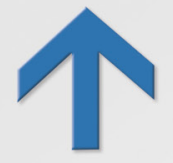

\section{CCHR}

\section{NOC}

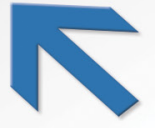

$\leftarrow$ EXIT 


\section{Critiques and Caveats}

A common critique of clinical decision rules is that they may not necessarily outperform subjective physician judgment, and that those who create these rules often do not explicitly compare their instruments against independent unassisted decision-making by clinicians..$^{2,3}$

Another drawback is that the misapplication of these rules can lead to increased testing, something particularly problematic for one-way rules, which only guide the provider in a single clinical direction. An example of a one-way rule is the Pulmonary Embolism Rule-Out Criteria (PERC), which advises that a low-risk patient who does not have any of the PERC factors will not require any further testing. This, however, does not necessarily mean that further testing is indicated in patients who have one of the PERC factors present. ${ }^{2}$ Thus, applying PERC and other one-way decisionmaking rules in a two-way fashion can prompt testing that would not be ordered based on clinician gestalt. Rules that are designed to help determine when testing is necessary and when it is unnecessary are referred to as two-way rules, an example of which is the Ottawa Ankle Rule.

Controversies aside, the incorporation of clinical decision rules in the electronic medical record of many institutions and the proliferation of smartphone applications utilizing these instruments have further cemented their place in EM. This article describes the more commonly used ED clinical decision rules, as well as pearls and pitfalls pertaining to their use. Part 1 that follows covers important validated rules related to trauma patients in the ED. Part 2, which will appear in an upcoming 2018 issue, will cover nontrauma medicaldiagnosis decision rules, including pulmonary embolism, and pneumonia.

\section{Head Trauma}

The increased utilization of computed tomography (CT) studies to assess for minor blunt head trauma spurred the develop- ment of clinical decision rules. In adult patients, the most popular and well-studied instruments are the New Orleans Criteria (NOC), the Canadian CT Head Rule (CCHR), and the National Emergency XRadiography Utilization Study (NEXUS) CT Head Rule.

\section{New Orleans Criteria}

The NOC validation cohort examined over 900 cases at a single trauma center, enrolling all patients 3 years of age and older who had suffered minor head trauma (defined as loss of consciousness (LOC) in a patient with grossly normal neurological examination and a Glasgow Coma Scale [GCS] score of 15), in the preceding 24 hours. ${ }^{4}$ Patients who experienced no LOC, had focal neurological deficit (except isolated short-term memory deficits), or who did not have any CT study performed, were excluded.

The NOC describes seven factors for consideration:

- Short-term memory deficits;

- Intoxication with drugs or alcohol;

- Physical evidence of trauma above the clavicles;

- Patients older than age 60 years;

- Seizure;

- Headache; and

- Vomiting.

The presence of at least one of these factors was found to be $100 \%$ sensitive and $25 \%$ specific for the presence of any traumatic intracranial abnormality on CT, though only $6.5 \%$ of patients in the derivation and validation cohorts had positive CT scans, and ultimately less than 1\% had lesions that required surgery. ${ }^{4}$

\section{Canadian CT Head Rule}

The CCHR validation study assessed over 2,700 patients at nine Canadian EDs, enrolling all patients aged 16 years and older who sustained a blunt head trauma less than 24 hours prior to presentation and who had a GCS score of 13 or higher. The investigators of the CCHR study specified that included patients should have suffered a witnessed 
LOC, definite amnesia, or a witnessed disorientation. ${ }^{5}$ Patients who did not have any of these factors were deemed to have minimal head trauma and were excluded from the study. Also excluded from this study were patients who had seizure prior to ED arrival, had a coagulopathy or used oral anticoagulants, had acute focal neurological deficit or obvious depressed skull fracture, had unstable vitals associated with a major trauma, or were pregnant. ${ }^{5}$ The CCHR was not designed to be applied to these excluded populations. Of note, patients with drug and alcohol intoxication were included in their validation. ${ }^{6}$

The CCHR describes five high-risk factors that increase the likelihood of requiring acute neurosurgical intervention:

- A GCS score of less than 15 at 2 hours after injury;

- Suspected open or depressed skull fracture;

- Any sign of basal skull fracture (eg, hemotympanum, raccoon eyes, cerebrospinal fluid otorrhea/rhinorrhea, Battle's sign;

- Two or more episodes of vomiting; or

- Patients aged 65 years or older.

In addition to these five high-risk factors, the CCHR also describes two medium risk criteria for finding any traumatic lesion on CT that would not necessitate acute neurosurgical intervention: amnesia of greater than 30 minutes before impact; and injury resulting from a dangerous mechanism such as a pedestrian struck by motor vehicle, occupant ejected from a motor vehicle, or a fall from a height greater than 3 feet or from over 5 stair-steps.

The presence of any one or more of the five high-risk factors was $100 \%$ sensitive for predicting the need for neurosurgical intervention, and taken together, having one or more of the seven factors was $100 \%$ sensitive for predicting clinically important brain injury. ${ }^{5}$

\section{NEXUS CT Head Rule}

The NEXUS CT Head Rule validation cohort included over 11,000 pediatric and adult blunt head trauma patients undergoing CT imaging at four hospital EDs, and excluded patients with penetrating injuries or presentation greater than 24 hours after injury. ${ }^{7}$ Patients were considered to be low risk if none of the following criteria were present:

- Age 65 years or older;

- Evidence of significant skull fracture;

Scalp hematoma;

- Neurological deficit;

- Altered level of alertness;

- Abnormal behavior;

- Coagulopathy; and

- Recurrent vomiting.

Patients who have one or more of these factors were considered as high risk. The presence of one of these factors was 100\% sensitive for detecting patients with lesions requiring neurosurgery and 99\% sensitive for detecting any significant intracranial injury, with specificity for either condition at $25 \% .^{7}$

\section{Decision-Rule Sensitivity Comparison}

Though the CCHR, NOC, and NEXUS rules differ in their inclusion/exclusion criteria, they have been compared to each other in different populations in the medical literature. A subgroup of the CCHR validation cohort and an accompanying external validation study of over 3,000 patients both found that the CCHR and the NOC were at least as sensitive $(100 \%)$ in detecting lesions requiring neurosurgical intervention, but the CCHR was more specific and had greater potential to reduce imaging rates. ${ }^{5,8}$ Another study examined the performance of NEXUS, CCHR, NOC, and other decision instruments in a database of nearly 8,000 adolescent and adult head trauma patients. The authors of this study found the three rules to have similarly high sensitivities ( $97 \%$ to $99 \%$ ) for detecting clinically important findings, but felt NEXUS to have the best combination of sensitivity and specificity compared to CCHR and NOC. ${ }^{9}$

Comment: A decision rule to decrease CT utilization in intoxicated head trauma

Continued on page 544 
Continued from page 541

patients is particularly useful, but only the CCHR can help potentially avoid imaging a drug or alcohol-intoxicated patient with abnormal behavior or altered level of alertness. Similarly, distinguishing superficial scalp trauma from more worrisome signs of intracranial injury is important, but the NOC and NEXUS rules recommend imaging for any trauma above the clavicles and for scalp hematomas.

Though the rules have similar sensitivities, the CCHR appears to have the greatest potential for aiding clinical decision-making. Of note, all of the adult decision rules consider patients older than age 60 or 65 years to be a high-risk feature. An instrument with better specificity for geriatric patients would be immensely helpful as our population continues to age.

The Pediatric Population: The PECARN Rule The CCHR, NEXUS, and NOC have been variously applied to pediatric populations, and the NEXUS and NOC included children (patients aged 3 years and older in the NOC) in their validation cohorts. Perhaps the most widely utilized validated rule for pediatric head trauma in the United States is the Pediatric Emergency Care Applied Research Network (PECARN) rule.

The PECARN rule is an age-specific instrument for assessing pediatric blunt head trauma patients for clinically important traumatic brain injury (TBI)-ie, brain injuries resulting in death, neurosurgery, intubation longer than 24 hours, or hospital admission length of more than two nights. ${ }^{10}$ The PECARN algorithm as described in its validation study is reproduced in Figure 1.

Patients with trivial injury mechanisms such as ground level falls or running into stationary objects and no signs or symptoms of head trauma other than scalp abrasions or lacerations were excluded from the PECARN analysis, as were patients with pre-existing neurological disorders, including ventricular shunts and brain tumors, patients with penetrating trauma, and those with bleeding disorders. The validation study of over 8,000 children found the presence of any of the criteria to be $100 \%$ and $96.8 \%$ sensitive in detecting clinically important TBI in children aged less than 2 years and children aged 2 years or older, respectively.

The PECARN rule was recently assessed in a large prospective cohort against two less extensively studied clinical decision rules: the Children's Head Injury Algorithm for the Prediction of Important Clinical Events (CHALICE) and the Canadian Assessment of Tomography for Childhood Head Injury (CATCH). Though the sample size was over 20,000 patients, only less than $1 \%$ required neurosurgery or died. The PECARN rule was determined to have the highest validation sensitivities $(100 \%$ for $<2$ years, $99 \%$ for $\geq 2$ years) of all of the rules; however, it has been noted by some clinicians that the strict application of the PECARN rule to the study population would have increased the rate of CT scanning 5-fold without providing any clear benefit over clinical judgment in detecting injury. ${ }^{11,12}$

Comment: The PECARN tool is by far the most robust instrument for pediatric head trauma, but striking a balance between finding otherwise clinically occult injury and reducing unnecessary testing/irradiation remains difficult in this vulnerable population.

\section{Cervical Spine Trauma}

Though the incidence of serious cervical spine injury in blunt trauma is low, the potentially devastating consequences of a missed lesion is a potent driver of radiographic testing. ${ }^{13}$ The Nexus Criteria and Canadian C-Spine Rule were developed to assist the clinician in determining when radiographic imaging is indicated in patients presenting with a blunt-traumarelated injury. The NEXUS criteria were developed to decrease cervical spine X-ray use based on five low-risk factors:

- No posterior midline tenderness; n No focal neurological deficit; 
Patients Aged $<2$ Years

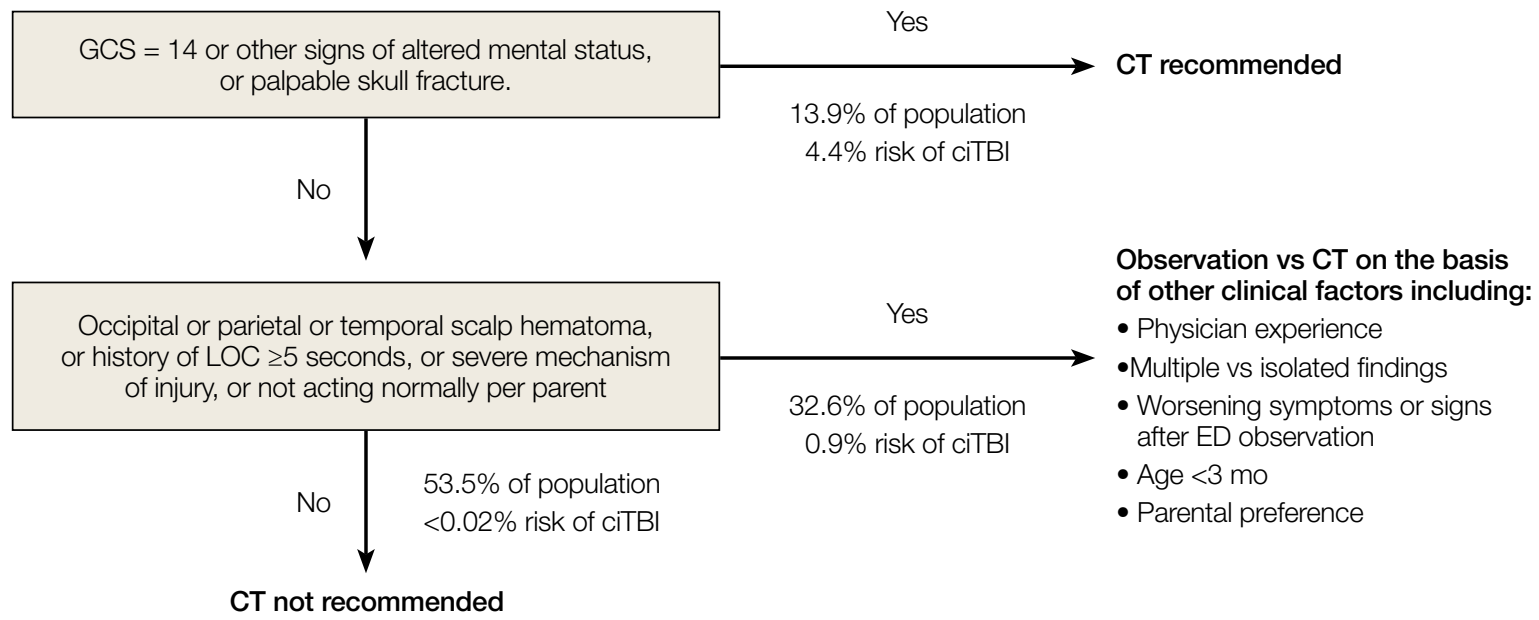

Patients Aged $\geq 2$ Years

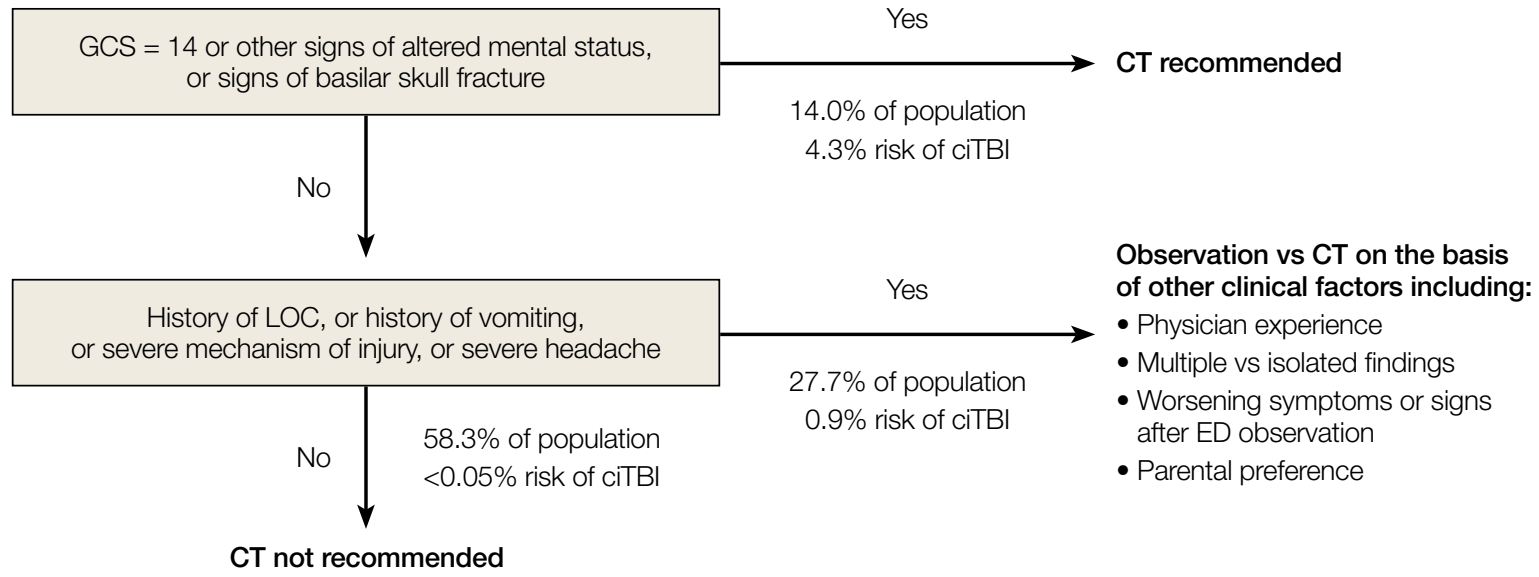

Figure 1. Algorithm illustrating application of the Pediatric Emergency Care Applied Research Network Rule.

Abbreviations: ciTBI, clinically important traumatic brain injury; CT, computed tomography; GCS, Glasgow Coma Scale; LOC, loss of consciousness.

normal level of alertness;

- No evidence of intoxication; and

- No clinically apparent pain that could

distract the patient from the pain of a cervical spine injury. ${ }^{13}$

Patients meeting these low-risk criteria were considered to not have a clinically significant cervical spine injury. Of note, the NEXUS investigators deemed several isolated radiographic lesions to be clinically insignificant in their analysis, including spinous process fractures, simple wedgecompression fractures without loss of $25 \%$ or more of vertebral body height, isolated avulsion fractures without ligamentous injury, type 1 odontoid fractures, transverse process fractures, end-plate fractures, trabecular bony injuries, and osteophyte fractures, except corner or teardrop fractures. ${ }^{13}$

The NEXUS validation study included 34,000 blunt trauma patients, including 3,000 patients aged 1 to 17 years and 
nearly 3,000 elderly patients, all of whom received at least 3-view X-rays (cross-table lateral, anteroposterior view, and openmouth odontoid view) unless CT or magnetic resonance imaging was performed because X-rays were deemed impractical or impossible..$^{13-15}$

The five low-risk features of NEXUS had a sensitivity of $99 \%$ in excluding clinically significant cervical spine trauma, and the authors determined that X-rays could have been avoided in more than $12 \%$ of the study population.

The NEXUS criteria have been criticized as being less reliable at the extremes of age, and the study authors have specifically urged caution in the use of this rule for infants and toddlers given the small number of these patients in the validation cohort. ${ }^{14}$

There have been multiple case reports and studies suggesting that the NEXUS rule does not perform well in elderly patients. ${ }^{15,16}$ Recent research has suggested that substituting "deviation from baseline mental status" for "normal level of alertness" and signs of head and neck trauma for distracting injury may improve specificity for detecting clinically significant injuries in geriatric fall patients, though the sample size does not approach the numbers from the original validation cohort. ${ }^{15,17}$

\section{Canadian C-Spine Rule}

The Canadian C-spine Rule (CCR), described in Figure 2, is more complex than the NEXUS criteria, but there are data to suggest it performs better in head-to-head evaluations. ${ }^{18}$ The validation cohort of CCR enrolled over 8,000 alert stable patients aged 16 years and older who had acute trauma to the head or neck presenting with either neck pain or no neck pain but visible injury above the clavicles, were nonambulatory, and had a dangerous mechanism of injury. Patients with a GCS of less than 15, unstable vitals, known vertebral disease, pregnancy, paralysis, or penetrating mechanism of injury were excluded.
Patients were assessed primarily on the basis of the three-view X-rays used in the NEXUS validation, however, only $71.7 \%$ of patients received radiographs and the remainder of patients were assessed using a telephone survey the authors had created during the derivation phase. ${ }^{18}$ Some lesions were deemed to be clinically unimportant, including isolated osteophyte avulsion, isolated fracture of a transverse process not involving a facet joint, isolated fracture of a spinous process not involving the lamina, or simple compression fracture involving less than $25 \%$ of the vertebral body height. ${ }^{18}$

Unfortunately, the CCR validation study was limited by incomplete evaluation in $10 \%$ of cases, though multiple subgroup analyses consistently demonstrated the CCR was 95\% sensitive or higher for detecting clinically important cervical spine injury, outperforming NEXUS. Subsequently, a large systematic review of 15 studies shows sensitivity of the CCR to be $90 \%$ to $100 \%$ and NEXUS to be $83 \%$ to $100 \% .^{19}$

Comment: The NEXUS criteria were validated in a larger and more heterogeneous cohort than CCR, and also have the advantage of being easier to remember. Using either rule, clinicians must consider that "clinically unimportant" cervical spine fractures are not excluded. Ultimately, the landscape of cervical spine assessment has shifted to performing $C T$ over plain radiographs, and these rules should be re-evaluated in this context. ${ }^{20}$

\section{Blunt Chest Trauma}

\section{NEXUS Chest Guidelines}

The NEXUS chest guidelines are more recent developments to help assess the need for chest imaging in the patient presenting with a blunt trauma. The first rule derived and validated by the investigators examined the utility of seven clinical criteria in predicting the need for chest imagingeither X-ray or CT:

- Patients older than age 60 years;

- Rapid deceleration mechanism defined as fall greater than 20 feet or motor vehicle crash greater than $40 \mathrm{mph}$; 


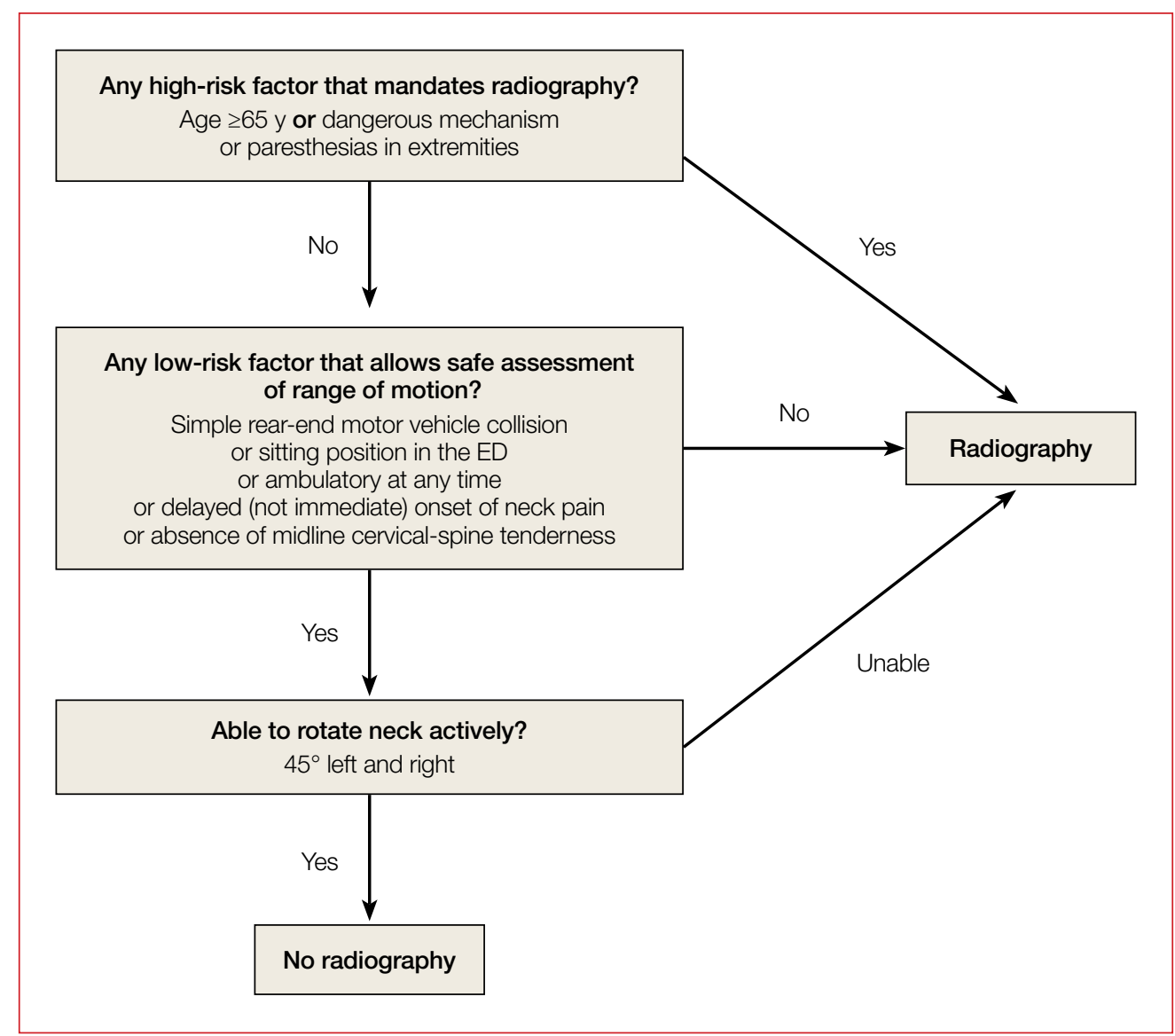

Figure 2. Algorithm illustrating application of the Canadian C-spine Rule.

Chest pain;

Intoxication;

- Abnormal alertness/mental status;

- Painful distracting injury; and

- Chest wall tenderness to palpation, with the exception of isolated clavicular tenderness to palpation. ${ }^{21}$

Of note, pericardial tamponade and cardiac contusion were not studied as they "are not primarily diagnosed by chest Xray (CXR) or chest CT." ${ }^{11}$ The NEXUS chest validation cohort included over 9,000 blunt trauma patients older than age 14 years receiving a variety of imaging modalities, but $43 \%$ received only a single CXR. The presence of one or more of the criteria had a sensitivity of $98.8 \%$ for detecting any traumatic injury on chest imaging, as well as a sensitivity of $99.7 \%$ for any major injury.

\section{CT-All Rule and CT-Major Rule}

Subsequently, the investigators of the NEXUS chest rule focused on creating a decision rule to decrease chest CT utilization in blunt trauma, as they found chest CT after a normal CXR to be lowyield. ${ }^{22}$ After classifying injuries as major or minor based on the necessity of procedural intervention, the authors derived two rules: CT-All, designed to not miss any injuries; and CT-Major, to identify major injuries requiring procedural intervention.

Hemothorax, pneumothorax, pneumomediastinum, or pulmonary contusion that were found on CT but did not require inpatient observation, intervention, or mechanical ventilation were considered clinically insignificant. ${ }^{23}$ 

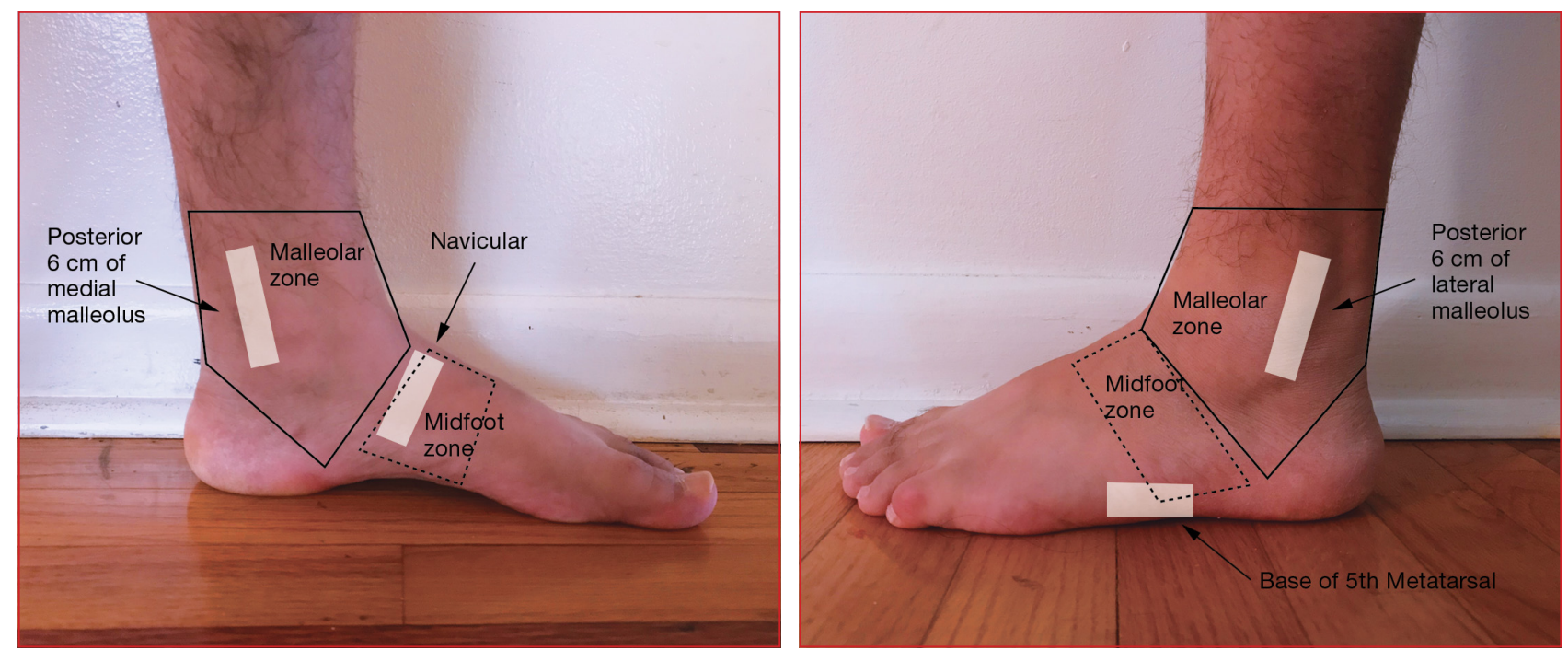

Figure 3. Photos outlining the anatomic landmarks for the Ottawa Ankle and Foot Rule.

The CT-Major includes six factors:

- Abnormal CXR showing any thoracic injury including clavicle fracture or widened mediastinum;

- Distracting injury;

- Any chest wall tenderness;

- Sternum tenderness;

- Thoracic spine tenderness; or

- Scapular tenderness.

The CT-All includes all of the CT-Major criteria and the additional criteria of rapid deceleration mechanism as defined previously. The validation cohort included over 5,000 patients aged 14 years and older. Having one or more of the major criteria was found to be $99.2 \%$ sensitive for major injury, and meeting one or more of the CTAll criteria were found to be $95.4 \%$ sensitive for any clinically significant injury. Both CT-Major and CT-All rules identified all 21 aortic/great vessel injuries in their study; the authors assert that vigilance for these types of injuries is often the primary justification for ordering CT imaging. ${ }^{23}$

Comment: Given the ubiquity and utility of the CXR, it seems unlikely that one would forgo a simple film on the basis of these rules. The presence of an abnormal CXR was by far the best screening criterion for major injury seen on subsequent chest CT, with sensitivity and specificity of $74.7 \%$ and $83.9 \%$, respectively. ${ }^{24}$ Of note, focused assessment with sonography for trauma (FAST) or eFAST (extended FAST) examinations were reported in only $63 \%$ or fewer patients in the derivation phase, so the rules did not incorporate point of care ultrasound findings as a criterion despite the growing influence of this modality on trauma decision-making. ${ }^{23}$

\section{Knee Trauma}

\section{Ottawa Knee Rules}

The Ottawa Knee Rules state that X-rays are only indicated if patients present with the following:

- Age older than 55 years;

- Isolated tenderness of the patella or fibular head;

- Inability to flex the knee to $90^{\circ}$; or

- Inability to bear weight (limping is allowed) for four steps both immediately following injury and at the ED.

In a prospective validation cohort of 1,096 adult patients, the rule was found to be $100 \%$ sensitive for detecting clinically important fractures-defined as any bone fragment at least $5 \mathrm{~mm}$ or avulsion if associated with complete disruption as tendons or ligaments-with a potential reduction Continued on page 550 
Continued from page 548

in radiography use by $28 \% .{ }^{25}$ Though the rule was designed for adult patients, it was validated in 750 children aged 2 to 16 years and found to be $100 \%$ sensitive with a $31 \%$ potential reduction in radiographs. ${ }^{26}$

\section{Pittsburgh Knee Rules}

The Pittsburgh Knee Rules only apply to patients with blunt trauma or fall to the knee-not twisting injury-and state $\mathrm{X}$ rays are indicated if patients are younger than age 12 years or older than age 50 years, and are unable to bear weight fully on toe pads and heels for four steps (limping is not allowed).

An initial validation of 133 patients demonstrating $100 \%$ sensitivity was followed by a larger external validation study. ${ }^{27}$ That study prospectively applied the Pittsburgh and the Ottawa rules in over 700 patients and found the sensitivity and specificity of the rules to be $99 \%$ and $60 \%$ for Pittsburgh and $97 \%$ and $27 \%$ for Ottawa. ${ }^{28}$

Comment: The ability to apply a rule to "all-comers" is important to EPs. The Ottawa instrument is more broadly applicable and has even been assessed in pediatric populations.

\section{Foot and Ankle Trauma}

\section{Ottawa Foot and Ankle Rule}

The most well-studied clinical decision rule in this category is the Ottawa Foot and Ankle Rule. ${ }^{29}$ This rule states that an ankle $\mathrm{X}$-ray is only required if there is pain in the malleolar zone and bony tenderness to palpation at the posterior edge or tip of either the lateral or medial malleolus, or the inability to bear weight both immediately after injury and in the ED. The anatomic landmarks are shown in Figure 3. When assessing a patient for a midfoot fracture, a foot X-ray is required if any of the following is present:

- Pain in the midfoot zone and either tenderness at the base of the fifth metatarsal;

- Tenderness over the navicular; or

- Inability to bear weight both immediately after the injury and at the ED.
The prospective validation study included over 1,400 adult patients with blunt trauma or twisting injury within 10 days of presentation. Clinically significant fractures were defined as malleolar or midfoot fractures with bone fragments greater than $3 \mathrm{~mm}$. The rule was found to be $100 \%$ sensitive for detecting both ankle and midfoot fractures, and would lead to a reduction of radiographs by $34 \%$ for the ankle and $30 \%$ for the foot. ${ }^{29}$ These results have borne out in multiple studies including a recent meta-analysis comparing six different decision instruments where the Ottawa rule was found to be superior. ${ }^{30}$

Though the initial validation cohort of the Ottawa rule did not include pediatric patients, it has subsequently been applied to this population. The initial validation study in the pediatric population included 670 patients aged 2 to 16 years. In addition to the criteria for clinically significant injuries in the original validation study, Salter Harris type I fractures, though treated with immobilization, were not deemed to be clinically significant injuries in this cohort.

Although the Ottawa rules were determined to be $100 \%$ sensitive for detecting significant ankle and midfoot fractures in the pediatric cohort, one of the study sites experienced an increase rather than a decrease in X-rays when this rule was applied. ${ }^{31}$

\section{Low Risk Ankle Rule}

Investigators for the pediatric specific Low Risk Ankle Rule posited that many pediatric patients with mild ankle injury refuse to bear weight on the extremity. Also, the most common fracture among preadolescent patients is a Salter-Harris type I fracture of the distal fibular epiphysis, which the investigators felt was commonly a clinical diagnosis with little to be gained from X-ray. ${ }^{32}$ These factors would prompt clinicians using the Ottawa rule to order imaging that may not be necessary in pediatric patients.

The Low Risk Ankle Rule states that if a patient has a low-risk examination, which 
is defined as tenderness and swelling isolated to the distal fibula and/or adjacent lateral ligaments distal to the tibial anterior joint line, then X-rays may not be necessary to exclude a high-risk ankle injury. The anatomic landmarks are shown in Figure 4.

High-risk injuries were defined as any injury leading to an unstable ankle, and do not include avulsion, buckle, and nondisplaced Salter-Harris types I and II fractures of the distal fibula. In the validation study of nearly 600 children, the sensitivity for detecting a high risk fracture was $100 \%$ and $\mathrm{X}$-rays could have been reduced in $62.8 \%$ of children with low-risk examinations compared to only $12 \%$ with the Ottawa Ankle Rule.

Comment: Although the Low Risk Ankle Rule was shown to reduce radiographic imaging by almost $63 \%$, it omits many patients who would require splinting or subspecialty follow-up. Even the Salter-Harris type I fractures in the pediatric validation of the Ottawa rule were treated with splinting, though they were not regarded as "significant" injuries. Clinicians applying these rules, especially to a pediatric population, should have a good sense of what type of injuries these rules are designed to detect.

\section{Conclusion}

In the midst of a busy shift, clinical decision rules can help save time and expense. However, few of the rules described are meant to be applied to "all-comers," and practitioners should be careful to not apply these rules to populations that were excluded in the validation cohorts. While clinical decision rules can help identify high-risk features, they are not a substitute for performing a thorough history and physical examination. Further studies should focus on whether these rules truly outperform unaided clinical decision-making.

Part 2 of "Playing by the Rules" will examine the use of clinician decision rules for nontraumatic conditions.

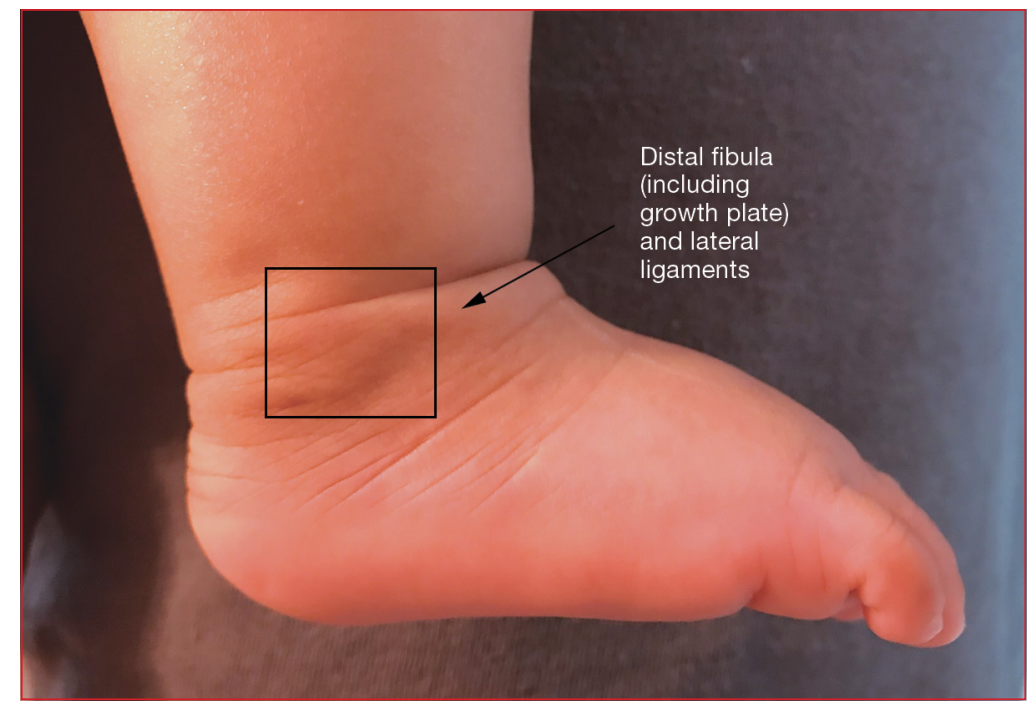

Figure 4. Photo outlining anatomic landmarks for the Low Risk Ankle Rule.

\section{References}

1. Stiell IG, Bennett C. Implementation of clinical decision rules in the emergency department. Acad Emerg Med. 2007;14(11):955-959. doi:10.1197/j. aem.2007.06.039.

2. Green SM. When do clinical decision rules improve patient care? Ann Emerg Med. 2013;62(2):132-135. doi:10.1016/j.annemergmed.2013.02.006.

3. Schriger DL, Elder JW, Cooper RJ. Structured clinical decision aids are seldom compared with subjective physician judgment, and are seldom superior. Ann Emerg Med. 2017;70(3):338-344.e3. doi:10.1016/j. annemergmed.2016.12.004.

4. Haydel MJ, Preston CA, Mills TJ, et al. Indications for computed tomography in patients with minor head injury. N Engl J Med. 2000;343(2):100-105. doi:10.1056/NEJM200007133430204.

5. Stiell IG, Clement CM, Rowe BH, et al. Comparison of the Canadian CT head rule and the New Orleans criteria in patients with minor head injury. JAMA. 2005;294(12):1511-1518. doi:10.1001/ jama.294.12.1511

6. Stiell IG, Perry JJ. Traumatic intracranial injury in intoxicate patients with minor head trauma. Acad Emerg Med. 2014;21(2):221. doi:10.1111/ acem. 12306.

7. Mower WR, Gupta M, Rodriguez R, Hendey GW. Validation of the sensitivity of the National Emergency X-Radiography Utilization Study (NEXUS) head computed tomographic (CT) decision instrument for selective imaging of blunt head injury patients: An observational study. PLoS Med. 2017;14(7):e1002313. doi:10.1371/journal.pmed.1002313.

8. Smits M, Dippel DW, de Haan GG, et al. External validation of the Canadian CT head rule and the New Orleans criteria for CT scanning in patients with minor head injury. JAMA. 2005;294(12):15191525. doi:10.1001/jama.294.12.1519.

9. Stein SC, Fabbri A, Servadei F, Glick HA. A critical comparison of clinical decision instruments for computed tomographic scanning in mild closed traumatic brain injury in adolescents and adults. Ann Emerg Med. 2009;53(2):180-188. doi:10.1016/j. annemergmed.2008.01.002. 
10. Kuppermann N, Holmes JF, Dayan PS, et al. Identification of children at very low risk of clinically-important brain injuries after head trauma: a prospective cohort study. Lancet. 2009;374(9696):1160-1170. doi:10.1016/S0140-6736(09)61558-0.

11. Babi FE, Borland ML, Phillips N, et al. Accuracy of PECARN, CATCH, and CHALICE head injury decision rules in children: a prospective cohort study. Lancet. 2017;389(10087):2393-2402. doi:10.1016/ S0140-6736(17)30555-X.

12. Mower WR. Paediatric head imaging decisions are not child's play. Lancet. 2017;389(10087):2354-2355. doi:10.1016/S0140-6736(17)30932-7.

13. Hoffman JR, Mower WR, Wolfson AB, Todd KH, Zucker MI. Validity of a set of clinical criteria to rule out injury to the cervical spine in patients with blunt trauma. National Emergency X-Radiography Utilization Study Group. N Engl J Med. 2000;343(2):94-99. doi:10.1056/NEJM200007133430203.

14. Viccellio P, Simon H, Pressman BD, Shah NM, Mower WR, Hoffman JR; NEXUS Group. A prospective multicenter study of cervical spine injury in children. Pediatrics. 2001;108(2):20.

15. Tran J, Jeanmonod D, Agresti D, Hamden K, Jeanmonod RK. Prospective validation of modified NEX US cervical spine injury criteria in low-risk elderly fall patients. West J Emerg Med. 2016;17(3):252-257. doi:10.5811/westjem.2016.3.29702.

16. Paykin G, O’Reilly G, Ackland HM, Mitra B. The NEXUS criteria are insufficient to exclude cervical spine fractures in older blunt trauma patients. Injury. 2017;48(5):1020-1024. doi:10.1016/j. injury.2017.02.013.

17. Evans D, Vera L, Jeanmonod D, Pester J, Jeanmonod R. Application of National Emergency X-Ray Utilizations Study low-risk c-spine criteria in high-risk geriatric falls. Am J Emerg Med. 2015;33(9):11841187. doi:10.1016/j.ajem.2015.05.031.

18. Stiell IG, Clement CM, McKnight RD, et al. The Canadian C-spine rule versus the NEXUS low-risk criteria in patients with trauma. $N$ Engl J Med. 2003;349(26):2510-2518

19. Michaleff ZA, Maher CG, Verhagen AP, Rebbeck T, Lin CW. Accuracy of the Canadian C-spine rule and NEXUS to screen for clinically important cervical spine injury in patients following blunt trauma: A systematic review. CMAJ. 2012;184(16):E867-E876. doi:10.1503/cmaj.120675.

20. Gale SC, Gracias VH, Reilly PM, Schwab CW. The inefficiency of plain radiography to evaluate the cervical spine after blunt trauma. J Trauma. 2005;59(5):1121-1125.
21. Rodriguez RM, Anglin D, Langdorf MI, et al. NEXUS chest: validation of a decision instrument for selective chest imaging in blunt trauma. JAMA Surg. 2013;148(10):940-946. doi:10.1001/jamasurg.2013.2757.

22. Rodriguez RM, Baumann BM, Raja AS, et al. Diagnostic yields, charges, and radiation dose of chest imaging in blunt trauma evaluations. Acad Emerg Med. 2014;21(6):644-650. doi:10.1111/acem.12396.

23. Rodriguez RM, Langdorf MI, Nishijima D, et al. Derivation and validation of two decision instruments for selective chest CT in blunt trauma: a multicenter prospective observational study (NEXUS Chest CT). PLoS Med. 2015;12(10):e1001883. doi:10.1371/journal.pmed.1001883.

24. Rodriguez RM, Hendey GW, Mower WR, et al. Selective chest imaging for blunt trauma patients: The national emergency X-ray utilization studies (NEXUS-chest algorithm). Am J Emerg Med. 2017;35(1):164-170. doi:10.1016/j.ajem.2016.10.066.

25. Stiell IG, Greenberg GH, Wells GA, et al. Prospective validation of a decision rule for the use of radiography in acute knee injuries. JAMA. 1996;275(8):611-615.

26. Bulloch B, Neto G, Plint A, et al. Validation of the Ottawa Knee Rule in children: a multicenter study. Ann Emerg Med. 2003;42(1):48-55. doi:10.1067/ mem.2003.196.

27. Seaberg DC, Jackson R. Clinical decision rule for knee radiographs. Am J Emerg Med. 1994;12(5): 541-543.

28. Seaberg DC, Yealy DM, Lukens T, Auble T, Mathias S. Multicenter comparison of two clinical decision rules for the use of radiography in acute, high-risk knee injuries. Ann Emerg Med. 1998;32(1):8-13.

29. Stiell IG, Greenberg GH, McKnight RD, et al. Decision rules for the use of radiography in acute ankle injuries. Refinement and prospective validation. JAMA. 1993;269(9):1127-1132.

30. Barelds I, Krijnen WP, van de Leur JP, van der Schans CP, Goddard RJ. Diagnostic accuracy of clinical decision rules to exclude fractures in acute ankle injuries: Systematic review and meta-analysis J Emerg Med. 2017;53(3):353-368. doi:10.1016/j. jemermed.2017.04.035

31. Plint AC, Bulloch B, Osmond MH, et al. Validation of the Ottawa Ankle Rules in children with ankle injuries. Acad Emerg Med. 1999;6(10):1005-1009.

32. Boutis K, Komar L, Jaramillo D, et al. Sensitivity of a clinical examination to predict need for radiography in children with ankle injuries: a prospective study. Lancet. 2001;358(9299):2118-2121. doi:10.1016/ S0140-6736(01)07218-X. 